\title{
A Description of Blowup for the Solid Fuel Ignition Model
}

\section{J. BEBERNES, A. BRESSAN \& D. EBERLY}

$\S 1$ Introduction. The purpose of this paper is to give a precise description of the asymptotic behavior of a radially symmetric solution $u(x, t)$ of

$$
u_{t}-\Delta u=e^{u}
$$

in a neighborhood of the blow-up point as $t$ approaches the finite blow-up time $T<\infty$ provided $x \in B_{R} \equiv\{x:|x|<R\} \subset \mathbf{R}^{n}$ and $n=1$ or 2 . Giga and Kohn [7] recently characterized the asymptotic behavior of the solution $u(x, t)$ of

$$
u_{t}-\Delta u=u^{p} \quad \Omega \subset \mathbf{R}^{n}
$$

near a blow-up singularity assuming a suitable upper bound on the rate of blowup, provided $n=1,2$ or $n \geq 3$ and $p \leq \frac{n+2}{n-2}$. For $\Omega=B_{R} \subset \mathbf{R}^{n}$, in light of recent a priori bounds established by Friedman and McLeod [6], this implies that the solution $u(x, t)$ of $(\mathrm{H})$ with suitable initial and boundary conditions satisfies

$$
(T-t)^{\beta} u(|x|, t) \rightarrow \beta^{\beta} \quad \text { as } t \rightarrow T^{-}
$$

provided $0 \leq|x| \leq C(T-t)^{1 / 2}$ for some $C$ and $\beta=\frac{1}{p-1}$.

For (I) we prove that the solution $u(x, t)$ satisfies

$$
u(x, t)-\ln \frac{1}{T-t} \rightarrow 0
$$

uniformly on $0 \leq|x| \leq C(T-t)^{1 / 2}, C>0$, as $t \rightarrow T^{-}$.

Equation (I) is the ignition period model for the thermal explosion of a nondeformable material of finite extent undergoing a single-step exothermic reaction. This model neglects the consumption of fuel and describes the temperature $u$ at any time and at any point in the bounded container. For $R>0$ sufficiently small, the temperature $u(x, t)$ becomes unbounded in the $L^{\infty}$-sense as $t$ approaches a finite blow-up time $T([1],[10],[4])$ and the blowup occurs at a single point, the center of the ball $B_{R}[6]$.

The main result of this paper is Theorem 4.6 which gives a precise description of how the blowup asymptotically behaves. The proof is valid only 
in dimensions 1 and 2. Two technical theorems-Theorems 3.1 and 4.5-are needed from which our main result follows immediately. Our proof of Theorem 3.1 is strongly influenced by the paper of Giga and Kohn, using critical a priori estimates due to Friedman and McLeod. But the real difficulty in understanding the blowup for (I) lies in determining the nonincreasing globally Lipschitz continuous solutions of the associated steady-state equation

$$
y^{\prime \prime}+\left(\frac{n-1}{\eta}-\frac{\eta}{2}\right) y^{\prime}+e^{y}-1=0
$$

on $[0, \infty)$, where $y^{\prime}(0)=0, y(0)=\alpha \geq 0$. For $n=1$ Bebernes and Troy [3] proved that the only such solution is $y \equiv 0$. The method used to prove Theorem 4.5 in this paper, valid for $n=1$ and 2, is based on ideas due to Eberly [5].

Kassoy-Poland [9] and Kapila [8] in earlier papers derived (K) from an asymptotic final time analysis of (I) and predicted on the basis of numerical calculations the existence of a solution of $(K)$ which satisfies the asymptotic condition $y(\eta) \sim-2 \ell \mathrm{n} \eta+K_{0}$ as $\eta \rightarrow \infty$. This implies the existence of a globally Lipschitz continuous solution of $(\mathrm{K})$. Our result shows that, for $n=1$ and 2, their final time analysis is incomplete. More precisely, we remark that: (i) their derivation of the asymptotic condition is incorrect, and (ii) the space variable in the inner expansion $\eta=\frac{x}{\sqrt{T-t}}$ is stretched too much so that no information concerning the spatial behavior of the solution is retained in the limit. This leaves open the problem of finding a different rescaling of the space coordinates for which in the limit a non-constant behavior of solutions can still be observed.

§2. Preliminaries. The nondimensional ignition model for a supercritical high activation energy thermal explosion of a solid fuel in a bounded container $\Omega$ can be described by

$$
\begin{aligned}
& u_{t}-\Delta u=e^{u} \\
& \begin{cases}u(x, 0)=\varphi(x) \geq 0, & x \in \Omega \\
u(x, t)=0, & x \in \partial \Omega, t>0\end{cases}
\end{aligned}
$$

where $\Omega=\left\{x \in \mathbf{R}^{n}:|x|<R\right\}$ and $\varphi$ is radially symmetric and decreasing $(\varphi(x) \geq \varphi(y) \geq 0$ for $|x| \leq|y| \leq R\})$ and $\Delta \varphi+e^{\varphi} \geq 0$ on $\Omega$.

We assume $R>0$ and $\varphi(x) \geq 0$ are such that the radially symmetric solution $u(x, t)$ blows up in finite time $T>0$. By the maximum principle $u(\cdot, t)$ is radially decreasing for $t \in[0, T)$ and $u_{t}(x, t) \geq 0$ for all $(x, t) \in \Gamma=\Omega \times[0, T)$.

Friedman and McLeod [6] recently proved that blowup occurs only at the origin $x=0$.

In addition, the results proven in [6] yield the following estimates: 
(2.3) For all $\alpha<1$, there exists $C_{\alpha}$ such that

$$
u(x, t) \leq \frac{2}{\alpha} \ln \frac{1}{|x|}+C, \quad(x, t) \in \Gamma .
$$

(2.4) There exists $\bar{t}<T$ such that

$$
|\nabla u(x, t)| \leq 2 e^{u(0, t) / 2} \quad \text { for } t \in[\bar{t}, T), x \in \bar{\Omega} .
$$

(2.5) There exists $\delta>0$ such that

$$
u_{t}(x, t) \geq \delta e^{u(x, t)}, \quad t \in\left[\frac{T}{2}, T\right), x \in\left[-\frac{R}{2}, \frac{R}{2}\right] .
$$

From (2.1) and (2.5), it follows that, for some $\delta>0, \delta e^{u(0, t)} \leq u_{t}(0, t) \leq$ $e^{u(0, t)}$ for $t \in\left[\frac{T}{2}, T\right)$, and hence

$$
-\ln (T-t) \leq u(0, t) \leq-\ln (T-t)-\ln \delta .
$$

§3. Final Time Analysis. Since the solution $u(x, t)$ of $(2.1)-(2.2)$ is radially symmetric, the initial-boundary value problem can be reduced to a problem in one spatial dimension.

Let $D=\{(r, t): 0 \leq t<T, 0 \leq r \leq R\}$. If $r=|x|$, then $v(r, t)=u(x, t)$ is well-defined on $D$ and satisfies:

$$
\begin{aligned}
v_{t} & =v_{r r}+\frac{n-1}{r} v_{r}+e^{v}, \\
v(r, 0) & =\varphi(r), \quad r \in[0, R], \\
v_{r}(0, t) & =0, v(R, t)=0, \quad t \in[0, T) .
\end{aligned}
$$

We wish to analyze the behavior of $v$ as $t \rightarrow T$. To do this we make the following change of variables:

$$
\tau=-\ln (T-t), \quad \eta=r(T-t)^{-1 / 2}, \quad \vartheta=v-\tau,
$$

whose inverse is

$$
t=T-e^{-\tau}, \quad r=\eta e^{-\tau / 2} .
$$

The domain $D$ transforms into $D^{\prime}$ with the change of variables above where

$$
D^{\prime}=\left\{(\eta, \tau): 0 \leq \eta \leq \operatorname{Re}^{\tau / 2}, \tau \geq-\ln T\right\}
$$

and $\vartheta=v-\tau$ solves the following initial-boundary value problem

$$
\begin{gathered}
\vartheta_{\tau}=\vartheta_{\eta \eta}+\left(\frac{n-1}{\eta}-\frac{\eta}{2}\right) \vartheta_{\eta}+e^{\vartheta}-1, \\
\vartheta(\eta,-\ell \mathrm{n} T)=\varphi\left(\eta \cdot T^{1 / 2}\right)+\ell \mathrm{n} T \\
\vartheta_{\eta}(0, \tau)=0, \quad \vartheta\left(\operatorname{Re}^{\tau / 2}, \tau\right)=-\tau .
\end{gathered}
$$

We can now characterize the asymptotic behavior of $\vartheta(\eta, \tau)$ as $\tau \rightarrow \infty$. 
Theorem 3.1. If there is a unique function $y(\eta)$ which is a nonincreasing globally Lipschitz continuous solution of the problem

$$
\begin{aligned}
& y^{\prime \prime}+\left(\frac{n-1}{\eta}-\frac{\eta}{2}\right) y^{\prime}+e^{y}-1=0 \\
& y^{\prime}(0)=0, \quad y(0)=\alpha \geq 0
\end{aligned}
$$

then, as $\tau \rightarrow \infty$, the solution $\vartheta(\eta, \tau)$ tends uniformly to $y(\eta)$ on compact subsets of $\mathbf{R}^{+}$.

Proof. We begin our proof by establishing some a priori estimates on $\vartheta(\eta, \tau)$. From (2.6) we have

$$
0 \leq \vartheta(0, \tau) \leq-\ell \mathrm{n} \delta, \quad \text { for } \tau \text { sufficiently large, }
$$

and from (2.4) and (2.6) we deduce

$$
\left|v_{r}(r, t)\right| \leq 2 e^{-1 / 2(\ln (T-t)+\ell \ln \delta)}=\frac{2}{\sqrt{\delta}} e^{\tau / 2}
$$

and hence

$$
\left|\vartheta_{\eta}(\eta, \tau)\right|=\left|v_{r} r_{\eta}\right|=\left|v_{r}\right| e^{-\tau / 2} \leq \frac{2}{\delta^{1 / 2}}
$$

for all $(\eta, \tau) \in D^{\prime}$ with $\tau \geq \tau^{\prime}$ suitably large. Combining (3.7) and (3.8), we obtain

$$
-\frac{2}{\delta^{1 / 2}} \eta \leq \vartheta(\eta, \tau) \leq-\ell n \delta
$$

for all $(\eta, \tau) \in D^{\prime}$ with $\tau$ sufficiently large.

We also observe that, by (2.3), there exists a constant $K>0$ such that $0 \leq$ $v(r, t) \leq K$ on $\left[\frac{R}{2}, R\right] \times[0, T)$. Thus, $v(r, t)$ satisfies the differential inequality $v_{t} \leq v_{r r}+\frac{n-1}{r} v_{r}+e^{K}$ and $v(r, t) \leq z(r, t)$ on $\left[\frac{R}{2}, R\right] \times[0, T]$, where $z(r, t)$ is the solution of

$$
\begin{gathered}
z_{t}=z_{r r}+\frac{n-1}{r} z_{r}+e^{K}, \\
z\left(\frac{R}{2}, t\right)=v\left(\frac{R}{2}, t\right), z(R, t)=0, \\
z(r, 0)=\varphi(r) .
\end{gathered}
$$

We conclude that

$$
z_{r}(R, t) \leq v_{r}(R, t) \leq 0 \quad \text { for } 0 \leq t \leq T
$$


and hence $v_{r}(R, t)$ is uniformly bounded on $0 \leq t<T$.

Now define $\vartheta^{\sigma}(\eta, \tau)=\vartheta(\eta, \tau+\sigma)$ as the function obtained by shifting $\vartheta$ in time by the amount $\sigma$. It will be shown that as $\sigma \rightarrow+\infty, \vartheta^{\sigma}(\eta, \tau)$ converges to a solution $y(\eta)$ of (3.5)-(3.6) uniformly on compact subsets of $\mathbf{R}^{+} \times \mathbf{R}$. It is equivalent to prove that given any sequence $\left\{\sigma_{i}\right\}, \sigma_{i} \rightarrow \infty$, there exists a subsequence $\left\{\sigma_{j}\right\}$ such that $\vartheta^{\sigma_{j}}$ converges to $y(\eta)$ uniformly on compact subsets of $\mathbf{R}^{+} \times \mathbf{R}$.

To prove this, let any monotone sequence $\left\{\sigma_{i}\right\}$ with $\sigma_{i} \rightarrow \infty$ be given and let $N$ be a positive integer. For $i$ large enough, the rectangle

$$
Q_{2 N}=\{(\eta, \tau): 0 \leq \eta \leq 2 N,|\tau| \leq 2 N\}
$$

lies in the domain of $\vartheta^{\sigma_{i}}$. Notice that the radially symmetric function $\widetilde{\vartheta}(\tilde{\eta}, \tau)=$ $\vartheta^{\sigma_{i}}(|\tilde{\eta}|, \tau)$ solves the parabolic equation

$$
\widetilde{\vartheta}_{\tau}=\Delta \widetilde{\vartheta}-\frac{1}{2}\langle\tilde{\eta}, \nabla \widetilde{\vartheta}\rangle+e^{\tilde{\vartheta}}-1
$$

on the cylinder $\Gamma_{2 N}=\left\{(\tilde{\eta}, \tau) \in \mathbf{R}^{n+1}:|\tilde{\eta}| \leq 2 N,|\tau| \leq 2 N\right\}$ with

$$
-\frac{4 N}{\delta^{1 / 2}} \leq \widetilde{\vartheta}(\tilde{\eta}, \tau) \leq-\ln \delta
$$

because of (3.9). By Schauder's interior estimates, all partial derivatives of $\widetilde{\vartheta}$ can be uniformly bounded in the sub-cylinder $\Gamma_{N}<\Gamma_{2 N}$. The same, of course, holds for $\vartheta^{\sigma_{i}}$. In particular, $\vartheta^{\sigma_{i}}, \vartheta_{\tau}^{\sigma_{i}}, \vartheta_{\eta \eta}^{\sigma_{i}}$ are uniformly Lipschitz continuous on $Q_{N} \subset Q_{2 N}$. Their Lipschitz constants depend on $N$ but not on $i$. By Ascoli's theorem, there exists a subsequence $\left\{\sigma_{j}\right\}, \sigma_{j} \rightarrow \infty$, and a function $\bar{\vartheta}$ such that $\vartheta^{\sigma_{j}}, \vartheta_{\tau}^{\sigma_{j}}, \vartheta_{\eta \eta}^{\sigma_{j}}$ converge to $\bar{\vartheta}, \bar{\vartheta}_{\tau}, \bar{\vartheta}_{\eta \eta}$, respectively, uniformly on $Q_{N}$. Repeating this construction for all $N$ and taking a diagonal subsequence, we can therefore conclude that

$$
\vartheta^{\sigma_{j}} \rightarrow \bar{\vartheta}, \quad \vartheta_{\tau}^{\sigma_{j}} \rightarrow \bar{\vartheta}_{\tau}, \quad \text { and } \vartheta_{\eta \eta}^{\sigma_{j}} \rightarrow \bar{\vartheta}_{\eta \eta}
$$

uniformly on every compact subset in $\mathbf{R}^{+} \times \mathbf{R}$. Clearly, $\bar{\vartheta}$ satisfies (3.3) with $\bar{\vartheta}_{\eta}(\eta, \tau) \leq 0$ for all $(\eta, \tau) \in \mathbf{R}^{+} \times \mathbf{R}, \bar{\vartheta}_{\eta}(0, \tau)=0$ for $\tau \in \mathbf{R}$, and $\left|\bar{\vartheta}_{\eta}(\eta, \tau)\right| \leq$ $2 \delta^{-1 / 2}$ because these relations hold for all $\vartheta^{\sigma_{j}}$.

We now prove that $\bar{\vartheta}$ is time--invariant. This will be accomplished by showing that $\bar{\vartheta}_{\tau} \equiv 0$. For the solution $\vartheta(\eta, \tau)$ of (3.3)-(3.4), define the energy functional by

$$
E(\tau)=\int_{0}^{\mathrm{Re}^{\tau / 2}}\left[\frac{\rho}{2} \vartheta_{\eta}^{2}-\rho\left(e^{\vartheta}-\vartheta\right)\right] d \eta
$$

where $\rho=\eta^{n-1} e^{-\eta^{2} / 4}$. Then multiplying (3.3) by $\rho \vartheta_{\tau}$ and integrating from 0 to $\operatorname{Re}^{\tau / 2}$, we have 


$$
\begin{aligned}
\int_{0}^{\mathrm{Re}^{\tau / 2}} \rho \vartheta_{\tau}^{2} d \eta & =\int_{0}^{\mathrm{Re}^{\tau / 2}} \rho \vartheta_{\tau}\left[\vartheta_{\eta \eta}+\left(\frac{n-1}{\eta}-\frac{\eta}{2}\right) \vartheta_{\eta}\right] d \eta \\
& +\int_{0}^{\mathrm{Re}^{\tau / 2}} \rho \vartheta_{\tau}\left(e^{\vartheta}-1\right) d \eta \\
& =\int_{0}^{\mathrm{Re}^{\tau / 2}} \vartheta_{\tau}\left(\rho \vartheta_{\eta}\right)_{\eta} d \eta+\int_{0}^{\mathrm{Re}^{\tau / 2}} \frac{\partial}{\partial \tau}\left[\rho\left(e^{\vartheta}-\vartheta\right)\right] d \eta \\
& =-\int_{0}^{\mathrm{Re}^{\tau / 2}} \frac{\partial}{\partial \tau}\left[\frac{\rho}{2} \vartheta_{\eta}^{2}\right] d \eta+\left.\rho \vartheta_{\tau} \vartheta_{\eta}\right|_{0} ^{\mathrm{Re}^{\tau / 2}} \\
& +\int_{0}^{\mathrm{Re}^{\tau / 2}} \frac{\partial}{\partial \tau}\left[\rho\left(e^{\vartheta}-\vartheta\right)\right] d \eta
\end{aligned}
$$

Moreover, the time-derivative of $E$ is given by

$$
\begin{aligned}
\frac{d}{d \tau} E(\tau) & =\int_{0}^{\operatorname{Re}^{\tau / 2}}\left\{\frac{\partial}{\partial \tau}\left(\frac{\rho}{2} \vartheta_{\eta}^{2}\right)-\frac{\partial}{\partial \tau}\left(\rho\left(e^{\vartheta}-\vartheta\right)\right)\right\} d \eta \\
& +\frac{1}{2} \operatorname{Re}^{\tau / 2}\left\{\left(\operatorname{Re}^{\tau / 2}\right)^{n-1} e^{-\left(R^{2} e^{\tau}\right) / 4}\left[\frac{1}{2} \vartheta_{\eta}^{2}\left(\operatorname{Re}^{\tau / 2}, \tau\right)-e^{-\tau}-\tau\right]\right\}
\end{aligned}
$$

Therefore, for all $a, b$ with $-\ell \mathrm{n} T \leq a<b$, integrating (3.12) with respect to $\tau$ from $a$ to $b$ and using (3.13) yields

$$
\begin{aligned}
& \int_{a}^{b} \int_{0}^{\mathrm{Re}^{\tau / 2}} \rho \vartheta_{\tau}^{2} d \eta d \tau \\
& =\int_{a}^{b}-\frac{d}{d \tau} E(\tau) d \tau \\
& \quad+\int_{a}^{b}\left(\operatorname{Re}^{\tau / 2}\right)^{n-1} e^{-\left(R^{2} e^{\tau}\right) / 4} \vartheta_{\tau}\left(\operatorname{Re}^{\tau / 2}, \tau\right) \vartheta_{\eta}\left(\operatorname{Re}^{\tau / 2}, \tau\right) d \tau \\
& \quad+\frac{1}{2} \int_{a}^{b}\left(\operatorname{Re}^{\tau / 2}\right)^{n} e^{-\left(R^{2} e^{\tau}\right) / 4}\left[\frac{1}{2} \vartheta_{\eta}^{2}\left(\operatorname{Re}^{\tau / 2}, \tau\right)-e^{-\tau}-\tau\right] d \tau \\
& =E(a)-E(b)+\psi(a, b)
\end{aligned}
$$

Recalling that $\left|\vartheta_{\eta}\right| \leq 2 \delta^{-1 / 2}$ and observing that

$$
\vartheta_{\tau}\left(\operatorname{Re}^{\tau / 2}, \tau\right)=-\frac{1}{2} \operatorname{Re}^{-\tau / 2} v_{r}\left(R, T-e^{-\tau}\right)-1
$$


which is uniformly bounded as $\tau \rightarrow+\infty$ because $v_{r}(R, t)$ is uniformly bounded by $(3.10)$, we conclude that

$$
\lim _{a \rightarrow \infty}\left\{\sup _{b>a} \psi(a, b)\right\}=0 .
$$

For any fixed $N$, we shall prove that

$$
\iint_{Q_{N}} \rho \bar{\vartheta}_{\tau}^{2} d \eta d \tau=\lim _{\sigma_{j} \rightarrow \infty} \iint_{Q_{N}} \rho\left(\vartheta_{\tau}^{\sigma_{j}}\right)^{2} d \eta d \tau=0 .
$$

Note that it is not a restriction to assume that $\lim _{j \rightarrow \infty}\left(\sigma_{j+1}-\sigma_{j}\right)=+\infty$. For all $j$ large enough, $N \leq \operatorname{Re}^{\left(\sigma_{j}-N\right) / 2}$ and $\sigma_{j+1}-\sigma_{j} \geq 2 N$. Hence,

$$
\begin{aligned}
\int_{-N}^{N} \int_{0}^{N} \rho\left(\vartheta_{\tau}^{\sigma_{j}}\right)^{2} d \eta d \tau & \leq \int_{-N}^{-N+\sigma_{j+1}-\sigma_{j}} \int_{0}^{\mathrm{Re}^{\left(\tau+\sigma_{j}\right) / 2}} \rho\left(\vartheta_{\tau}^{\sigma_{j}}\right)^{2} d \eta d \tau \\
& =E\left(\sigma_{j}-N\right)-E\left(\sigma_{j+1}-N\right)+\psi\left(\sigma_{j}-N, \sigma_{j+1}-N\right)
\end{aligned}
$$

by (3.14). As a consequence of (3.15), we have

$$
\iint_{Q_{N}} \rho \bar{\vartheta}_{\tau}^{2} d \eta d \tau \leq \limsup _{j \rightarrow \infty}\left\{E\left(\sigma_{j}-N\right)-E\left(\sigma_{j+1}-N\right)\right\} .
$$

Fix any $K$ arbitrarily large. For $j$ sufficiently large, we have

$$
\begin{aligned}
& +\int_{0}^{K} \frac{\rho}{2}\left\{\left[\vartheta_{\eta}^{\sigma_{j}}(\eta,-N)\right]^{2}-\left[\vartheta_{\eta}^{\sigma_{j+1}}(\eta,-N)\right]^{2}\right\} d \eta \\
& -\int_{0}^{K} \rho\left\{\left[e^{\vartheta^{\sigma_{j}}(\eta,-N)}-\vartheta^{\sigma_{j}}(\eta,-N)\right]-\left[e^{\vartheta^{\sigma_{j+1}}(\eta,-N)}-\vartheta^{\sigma_{j+1}}(\eta,-N)\right]\right\} d \eta \\
& +\int_{K}^{\mathrm{Re} e^{\left(\sigma_{j}-N\right) / 2}} \rho\left\{\frac{1}{2}\left(\vartheta_{\eta}^{\sigma_{j}}(\eta,-N)^{2}-\left(e^{\vartheta^{\sigma_{j}}(\eta,-N)}-\vartheta^{\sigma_{j}}(\eta,-N)\right)\right\} d \eta\right. \\
& -\int_{K}^{\mathrm{Re}{ }^{\left(\sigma_{j}-N\right) / 2}} \rho\left\{\frac{1}{2}\left(\vartheta^{\sigma_{j+1}}(\eta,-N)\right)^{2}-\left(e^{\vartheta^{\sigma_{j+1}}(\eta,-N)}-\vartheta^{\sigma_{j+1}}(\eta,-N)\right)\right\} d \eta .
\end{aligned}
$$

In (3.17), the first two integrals on the right-hand side converge to zero as $j \rightarrow \infty$. Recalling that $\left|\vartheta_{\eta}^{\sigma_{j}}(\eta,-N)\right| \leq \frac{2}{\sqrt{\delta}}$ and $-\frac{2}{\sqrt{\delta}} \eta \leq \vartheta^{\sigma_{j}}(\eta,-N) \leq-\ell \mathrm{n} \delta$, we see that the sum of the absolute values of the last two integrals is bounded by

$$
2 \int_{K}^{\infty} \eta^{n-1} e^{-\eta^{2} / 4}\left(\frac{1}{2} \cdot \frac{4}{\delta}+\frac{1}{\delta}+\max \left\{-\ln \delta, \frac{2}{\sqrt{\delta}} \eta\right\}\right) d \eta
$$

which can be made arbitrarily small by choosing $K$ large enough. 
This proves that $\int_{-N}^{N} \int_{0}^{N} \rho \bar{\vartheta}_{\tau}^{2} d \eta d \tau=0$ and hence $\bar{\vartheta}_{\tau} \equiv 0$ and $\bar{\vartheta}$ is timeindependent. In particular, $\bar{\vartheta}(\eta, \tau)=\bar{\vartheta}(\eta, 0) \equiv y(\eta)$ which is the unique globally Lipschitz continuous nonincreasing solution of (3.5)-(3.6) with $y(0)=\alpha \in$ $[0,-\ln \delta]$.

$\S 4$. The Steady State Solution. In this section we prove that, in dimensions $n=1$ and 2 , the only nonincreasing globally Lipschitz continuous (GLC) solution $y(\eta)$ of the steady state problem $(3.5)-(3.6)$ is $y(\eta) \equiv 0$.

Lemma 4.1. Any nonincreasing nontrivial solution of (3.5)-(3.6) has a unique zero $\bar{x} \leq(2 n)^{1 / 2}$.

Proof. The linearized problem

$$
\begin{gathered}
u^{\prime \prime}+c(\eta) u^{\prime}=0 \\
u(0)=\alpha>0, \quad u^{\prime}(0)=0
\end{gathered}
$$

where $c(\eta)=\frac{n-1}{\eta}-\frac{\eta}{2}$ has a unique solution $u(\eta)=\alpha\left(1-\frac{\eta^{2}}{2 n}\right)$ with a unique positive zero at $z=(2 n)^{1 / 2}$. From (3.5), we have

$$
y^{\prime \prime}+c(\eta) y^{\prime}+y=-\left(e^{y}-y-1\right) \leq 0 .
$$

Let $w(\eta)=u y^{\prime}-u^{\prime} y$. Then $w^{\prime}+c(\eta) w \leq 0$ with $w(0)=0$. Thus $w(\eta) \leq 0$ for $\eta \geq 0$. Since $u>0$ on $[0, z),\left(\frac{y}{u}\right)^{\prime}=\frac{w}{u^{2}} \leq 0$ on $[0, z)$ and hence $y(\eta) \leq u(\eta)$ on $[0, z)$. Thus, $y(z) \leq u(z)$ and $y$ has a first zero $\bar{x} \leq z$ which is unique because $y$ is a nonincreasing nontrivial solution of (3.5).

Let $y(\eta)$ be a nonincreasing nontrivial solution of $(3.5)-(3.6)$ on $[0, \infty)$. Then define

$$
h(\eta)=y^{\prime \prime}+\frac{n-1}{\eta} y(\eta)=\eta^{1-n}\left(\eta^{n-1} y^{\prime}\right)^{\prime}
$$

Lemma 4.2. If $|h(\eta)| \geq \varepsilon>0$ for $\eta \geq \bar{x}$, then $y(\eta)$ is not globally Lipschitz continuous on $[0, \infty)$.

Proof. If $h(\eta) \leq-\varepsilon<0$ for $\eta \geq \bar{x}$, then $\left(\eta^{n-1} y^{\prime}\right)^{\prime} \leq-\varepsilon \eta^{n-1}$ and this implies $y^{\prime}(\eta) \leq k \eta^{1-n}-\frac{\varepsilon \eta}{n}$ for some constant $k$ and $\eta \geq \bar{x}$. Clearly $y$ cannot be GLC. Similarly, the same conclusion follows if $h(\eta) \geq \varepsilon>0$ for $\eta \geq \bar{x}$.

The function $h(\eta)$ defined by (4.3) satisfies

$$
\begin{gathered}
h^{\prime \prime}+c(\eta) h^{\prime}+\left(e^{y}-1\right) h=-e^{y}\left(y^{\prime}\right)^{2}, \quad 0<\eta<\infty \\
h(0)=1-e^{\alpha}, \quad h^{\prime}(0)=0 .
\end{gathered}
$$


Define $g(\eta)=\frac{\eta}{2} y^{\prime}(\eta)+1$. Then $g(\eta)$ satisfies

$$
g^{\prime}-\frac{\eta}{2} g=-\frac{\eta}{2} e^{y}+\frac{2-n}{2} y^{\prime}, \quad 0<\eta<\infty,
$$

and

$$
g^{\prime \prime}+c(\eta) g^{\prime}+\left(e^{y}-1\right) g=0
$$

with

$$
g(0)=1, \quad g^{\prime}(0)=0 .
$$

From (3.5), we also have

$$
h(\eta)=g(\eta)-e^{y(\eta)} .
$$

Lemma 4.3. There is an $\tilde{x}>0$ such that $g(\eta) \neq 0$ and $g^{\prime}(\eta) \neq 0$ for $\eta \geq \tilde{x}$.

Proof. Since $y^{\prime}(\eta) \leq 0$ for $\eta \geq 0$, we have that $g(\eta) \leq 1$ for all $\eta \geq 0$.

If $g$ has an unbounded sequence of zeros which are isolated by (4.7), then there must be an unbounded sequence $\left\{x_{k}\right\}$ of zeros of $g^{\prime}$ at which $g$ has local minima. There then is a first $k=m$ where $y\left(x_{m}\right)<0$ and $g\left(x_{m}\right)<0$. Then by (4.7), we have $g^{\prime \prime}\left(x_{m}\right)=\left(1-e^{y\left(x_{m}\right)}\right) g\left(x_{m}\right)<0$. This contradicts the fact that $g^{\prime}<0$ in a left neighborhood of $x_{m}$. Thus, $g$ cannot have an unbounded sequence of zeros. Let $x_{0}>0$ be such that $g(\eta) \neq 0$ for $x \geq x_{0}$.

Suppose $g(\eta)>0$ for $\eta \geq x_{0}$. If there were an unbounded sequence $\left\{x_{k}\right\}$ of zeros for $g^{\prime}$, then, again by $(4.7), g^{\prime \prime}\left(x_{k}\right)=\left[1-e^{y\left(x_{k}\right)}\right] g\left(x_{k}\right)>0$ for $k$ sufficiently large. This is impossible. A similar argument holds for $g(\eta)<0$ for $\eta \geq x_{0}$.

We conclude that there exists an $x_{1}>0$ such that $g^{\prime}(\eta) \neq 0$ for $\eta \geq x_{1}$. Let $\tilde{x}=\max \left(x_{0}, x_{1}\right)$. Then $g(\eta) \neq 0$ and $g^{\prime}(\eta) \neq 0$ for $\eta \geq \tilde{x}$.

Lemma 4.4. The function $g(\eta)$ has at least one zero.

Proof. Let $W(\eta)=g h^{\prime}-g^{\prime} h$, where $g(\eta)$ satisfies (4.7)-(4.8) and $h(\eta)$ satisfies (4.4)-(4.5). As long as $g(\eta)>0$, we have

$$
W^{\prime}+c(\eta) W=-e^{y}\left(y^{\prime}\right)^{2} g, \quad W(0)=0 .
$$

Thus,

$$
\begin{aligned}
W(\eta) & =-\eta^{1-n} e^{\eta^{2} / 4} \int_{0}^{\eta} s^{n-1} e^{-s^{2} / 4} e^{y(s)}\left(y^{\prime}(s)\right)^{2} g(s) d s \\
& =-\eta^{1-n} e^{\eta^{2} / 4} I(\eta),
\end{aligned}
$$

where $I(\eta)>0$. But $\left(\frac{h}{g}\right)^{\prime}=\frac{W}{g^{2}}$ so

$$
h(\eta)=\left(1-e^{\alpha}\right) g(\eta)-g(\eta) \int_{0}^{\eta} s^{1-n} e^{s^{2} / 4} I(s)[g(s)]^{-2} d s .
$$


Assume $g(\eta)$ has no zeros. If $g$ is bounded away from 0 , then there is an $\varepsilon>0$ such that $1 \geq g(\eta) \geq \varepsilon>0$ for $\eta>0$. By $(4.10)$, we have $h(\eta) \leq$ $\left(1-e^{\alpha}\right) \varepsilon=-k<0$. By definition of $h$, this implies $y^{\prime}(\eta) \leq-\frac{k \eta}{n}$ for $\eta \geq 0$. Thus, $g(\eta) \leq 1-\frac{k \eta^{2}}{n}$, which is impossible. Thus, the only way $g(\eta)$ cannot have a zero is for $\lim _{\eta \rightarrow \infty} g(\eta)=\lim _{\eta \rightarrow \infty} g^{\prime}(\eta)=0$ by Lemma 4.3 with $g(\eta)>0$. Applying L'Hôpital's rule in (4.10), we have that

$$
\begin{aligned}
\lim _{\eta \rightarrow \infty} h(\eta) & =-\lim _{\eta \rightarrow \infty} g(\eta) \int_{0}^{\eta} s^{1-n} e^{s^{2} / 4} I(s)[g(s)]^{-2} d s \\
& =\lim _{\eta \rightarrow \infty} \eta^{1-n} e^{\eta^{2} / 4} I(\eta)\left[g^{\prime}(\eta)\right]^{-1}=-\infty, .
\end{aligned}
$$

This contradicts Lemma 4.2. We conclude, $g(\eta)$ has at least one zero.

We now can prove the key result of this section.

Theorem 4.5. For $n=1$ or 2 , the only nonincreasing globally Lipschitz continuous solution $y(\eta)$ of $(3.5)-(3.6)$ is $y(\eta) \equiv 0$.

Proof. If $y(\eta)$ is any such nontrivial solution of (3.5)-(3.6), then $g(\eta)$, by Lemma 4.4, must have a first zero $\eta_{0}$. If there were an $\eta_{1}>\eta_{0}$ such that $g^{\prime}\left(\eta_{1}\right)=0$ and $g\left(\eta_{1}\right)<0$, then for $n=1$ or 2 , equation (4.6) implies that

$$
0<-\frac{1}{2} \eta_{1} g\left(\eta_{1}\right)=-\frac{1}{2} \eta_{1} e^{y\left(\eta_{1}\right)}+\frac{2-n}{2} y^{\prime}\left(\eta_{1}\right)<0 .
$$

This contradiction leads us to conclude that $g^{\prime}(\eta)<0$ for $\eta \geq \eta_{0}$. Hence $g(\eta) \leq-\varepsilon<0$ for $\eta \geq \tilde{x}>\eta_{0}$. By (4.9), we have $h(\eta) \leq-\varepsilon$ for $\eta \geq \tilde{x}$. By Lemma $4.2, y(\eta)$ cannot be GLC. This is a contradiction. We conclude that $y(\eta) \equiv 0$ is the only possible such solution.

As a consequence of Theorems 3.1 and 4.5, we have the following result.

Theorem 4.6. Let $n=1$ or 2 . As $t \rightarrow T^{-}$, the solution $v(r, t)$ of (3.1)-(3.2) satisfies

$$
v(r, t)-\ln (T-t)^{-1} \rightarrow 0
$$

uniformly on $0 \leq r \leq c(T-t)^{1 / 2}, c>0$.

\section{REFERENCES}

[1] J. BeBernes \& D. KASSOY, A mathematical analysis of blow-up for thermal reactions, SIAM J. Appl. Math. 40 (1981), 476-484.

[2] J. Bebernes \& D. KAssoy, Solution profiles and thermal runaway, Lectures in Applied Math., Amer. Math. Soc. 24 (1986), 217-228. 
[3] J. Bebernes \& W. Troy, Nonexistence for the Kassoy problem, SIAM J. Math. Anal. (to appear).

[4] H. Bellout, A criterion for blow-up of solutions to semilinear heat equations, (preprint).

[5] D. Eberly, Nonexistence for the Kassoy problem for dimensions 1 and 2, J. Math. Anal. Appl. (to appear).

[6] A. Friedman \& B. MCLeOd, Blow-up of positive solutions of semilinear heat equations, Indiana Univ. Math. Journal 34 (1985), 425-447.

[7] Y. GigA \& R. Kонn, Asymptotically self-similar blow-up of semilinear heat equations, Comm. Pure Appl. Math. XXXVIII (1985), 297-319.

[8] A. KAPIL A, Reactive-diffusive systems with Arrhenius kinetics: Dynamics of ignition, SIAM J. Appl. Math. 39 (1980), 21-36.

[9] D. KASSOY \& J. POLAND, The thermal explosion confined by a constant temperature boundary: I. The induction period solution, SIAM J. Appl. Math. 39 (1980), 412-430.

[10] A. LACEY, Mathematical analysis of thermal runaway for spatially inhomogenous reactions, SIAM J. Appl. Math. 43 (1983), 1350-1366.

This research was partially supported by the U. S. Army Research Office under contract number DAAG 29-85-K-0209.

\title{
J. BEBERNES \\ Department of Mathematics \\ University of Colorado \\ Boulder, Colorado 80309
}

\author{
A. BRESSAN \\ Istituto di Matematica Applicata \\ Università di Padova \\ 35100 Padova, Italy \\ D. EBERLY \\ Department of Mathematics \\ University of Texas \\ San Antonio, Texas 78285
}

Received November 15, 1985; revised January 16, $198 \%$. 